\title{
Transmission of 112(4x28)-Gb/s PAM-4 Signal over 48.6-km SSMF within Only 50-GHz Grid
}

\author{
Jian Dong ${ }^{1 *}$, Rong $\mathrm{Hu}^{2}$ \\ 1. School of Information Science and Engineering, Central South University, Changsha 410083, Hunan, China \\ 2. Wuhan Research Institute of Posts \& Telecommunications, Wuhan 430074, Hubei, China \\ Corresponding author: Jian Dong, dongjian@ mail.csu.edu.cn
}

\begin{abstract}
In this paper, a transmission of 112(4x28)-Gb/s PAM-4 signal is experimentally demonstrated within only 50-GHz grid, achieving an optical spectral efficiency (SE) of $2.24 \mathrm{~b} / \mathrm{s} / \mathrm{Hz}$. For the intensity modulation and direct detection (IM/DD) based PAM-4 transmission, it is the first time to the best of our knowledge that 112-Gb/s PAM-4 signal has been transmitted over 48.6-km standard single mode fiber (SSMF), which is compatible with $\mathbf{5 0 - G H z}$ standard gridding. By employing digital pre-equalization, duobinary encoding/decoding and 7-level based training sequence aided least-mean square (TS-LMS) algorithm, each lane of the 28-Gb/s PAM-4 signal occupies less than 11-GHz optical spectral (3-dB bandwidth), resulting in negligible inter-channel interference for the 4-lane 112-Gb/s PAM-4 signals within 50-GHz grid. The proposed method is bandwidth and computationally efficient, which is thought feasible in the low-cost short reach optical networks.
\end{abstract}

Index Terms - Intensity Modulation, Direct Detection, PAM-4.

\section{INTRODUCTION}

$\mathrm{D}$ UE to the fast growth of mobile and cloud services, there is strong demand for short reach optics with low cost and high capacity. The intensity modulation and direct detection (IM/DD) optics is well-suited to provide such low cost interfaces. With the IM/DD optics, various advanced modulation formats, such as 4-level pulse amplitude modulation (PAM-4), multi-band carrierless amplitude and phase modulation (Multi-CAP), discrete multi-tone modulation (DMT), have been proposed with up to 112-Gb/s per lane for data centers, PON or metro networks [1-8]. Among those candidates, the PAM-4 modulation, which is easy for signal generation and processing, has been under active study for standardization of $100 \mathrm{G}$ and $400 \mathrm{G}$ interfaces [9]. The demonstration of 100-Gb/s PAM-4 transmission over 30-km standard single mode fiber (SSMF) has been reported based on 10G-class TOSA/ROSA and electrical equalization [10], however, 4 channels are employed with 100-GHz spacing. To further increase the optical spectral efficiency (SE), Nyquist filtering is introduced to achieve higher capacity for each channel based on bandwidth limited optics. For instance, transmission of Nyquist shaped 56-Gb/s PAM-4 signal has been reported at 1550-nm window, in which 26-km reach is achieved by maximum likelihood sequence estimation (MLSE) [11]. And, N. Kikuchi et al. reported the generation of 102-Gb/s Nyquist PAM-4 signal based on commercial electro-absorptive modulated laser (EML) of $28-\mathrm{GHz}$ analog bandwidth [12].

Targeting at $112-\mathrm{Gb} / \mathrm{s}$ line rate within only $50-\mathrm{GHz}$ optical grid, the optical SE should be $2.24 \mathrm{~b} / \mathrm{s} / \mathrm{Hz}$. It is difficult to realize such a goal by traditional PAM-4 modulation or Nyquist shaping of PAM-4 signal at 56 GBaud/s. In this paper, partial response is first introduced by digital duobinary encoding of PAM-4 signal to achieve a SE of $2 \mathrm{~b} / \mathrm{s} / \mathrm{Hz}$ [13]. Then, an analog low pass filter (LPF) is used after the digital to analog convertor (DAC) to further compress the spectrum and increase the SE to >2.24 b/s/Hz. With the help of digital signal processing (DSP), digital channel pre-compensation is done in the frequency domain at transmitter to overcome the bandwidth limitation from electronics/optics. In the receiver DSP, a training sequence aided least-mean square (TS-LMS) algorithm is proposed to restore the 7-level signal. Then, duobinary decoding is carried out to convert the signal back to 4 levels. PAM-4 signal de-mapping and bit error ratio (BER) calculation comes lastly. Thanks to the 7-level training sequence, the proposed method outperformances significantly over traditional 4-level based CMMA method [14], when the optical SE is larger than $2 \mathrm{~b} / \mathrm{s} / \mathrm{Hz}$. In the experiment, 112(4x28)-Gb/s transmission of PAM-4 signal is demonstrated over 48.6-km SSMF within 50-GHz grid, in which 4 lanes are used considering the extended reach and lower bandwidth requirement of electronics/optics. The required sampling rate of $\mathrm{ADC} / \mathrm{DAC}$ is only $14 \mathrm{GSa} / \mathrm{s}$. Further spectrum shaping is realized by a $5.5-\mathrm{GHz}$ analog $\mathrm{LPF}$ after the DAC, resulting in less than 11-GHz bandwidth $(3-\mathrm{dB})$ for each lane. It is the first demonstration to the best of our knowledge that $112-\mathrm{Gb} / \mathrm{s}$ PAM-4 signal is transmitted over 48.6-km SSMF, still compatible with the 50-GHz gridding. The proposed method is both bandwidth and computationally efficient, which is thought feasible in the low-cost short reach optical networks.

\section{PRINCIPAL OF OPERATION}

Partial response is introduced by digital duobinary encoding of PAM-4 signal, which is fulfilled by a two-tap finite impulse 
response (FIR) filter with equal weights. Because the generation method introduces correlation between adjacent bits, meaning that the current bit is also defined by the values of the k preceding bits, pre-coding at the transmitter side is necessary in order to avoid error propagation at the receiver side. Fig. 1 shows the flow chart for duobinary encoding and decoding,

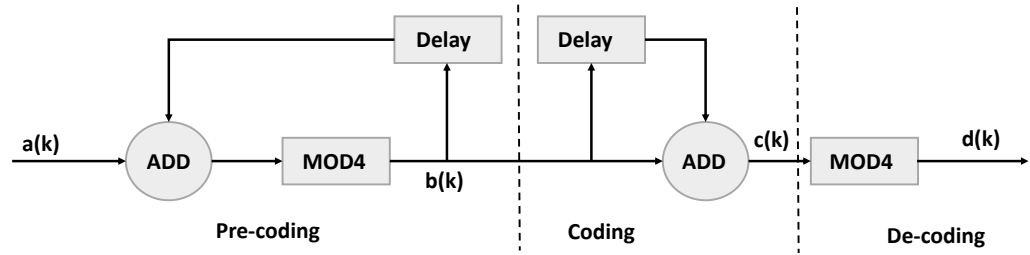

Fig. 1 Flow chart for procedures of duobinary encoding and decoding.

where $\mathrm{a}(\mathrm{k})$ is the original bit sequence, $\mathrm{b}(\mathrm{k})$ is the pre-coded PAM-4 sequence, and $\mathrm{c}(\mathrm{k})$ is the generated 7-level signal. It is possible to recover the PAM-4 sequence $\mathrm{d}(\mathrm{k})$ from independent decisions on $\mathrm{c}(\mathrm{k})$ by using simple 'mod4' operation.

The partial response PAM-4 signal can be also achieved with a digital Bessel low pass filter (LPF) [15], which however requires a blind channel equalization with constant multi-level modulus algorithm (CMMA) in the receiver DSP. While, a 7-level training sequence can be prepared for fast convergence of FIR weights with TS-LMS algorithm. Fig. 2 shows the spectrums of traditional PAM-4 signal and partial response PAM-4 signal by duobinary encoding, respectively. Note that the spectrum of duobinary encoded PAM-4 signal is only half of original signal, however, a LPF is required after the encoder for further spectrum shaping to increase the SE and void inter-channel interferences (ICI) in the multi-channel environments.

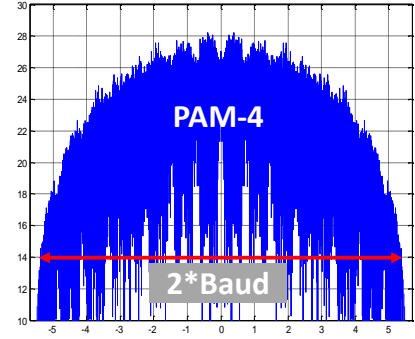

(a)

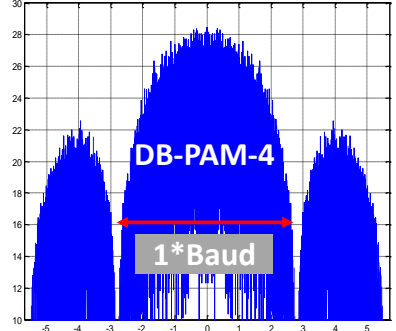

(b)

Fig. 2: Spectrum of (a) PAM-4 signal and (b) partial response (duobinary encoded) PAM-4 signal, respectively.

Another performance limiting factor comes from the limited bandwidth and nonlinear channel responses, which may include the modulator, driver, detector, and ADC/DAC. Fig. 3(a) and (b) shows the measured amplitude and phase response (S21 parameters) of the system. A sine wave like vibration is found in the $\mathrm{S} 21$ curves (> $2 \mathrm{~dB}$ for amplitude response at $7 \mathrm{GHz}$ ), which is mainly due to the impairment from ADC/DAC. The overall 3-dB bandwidth is only $3 \mathrm{GHz}$. Thus, channel pre-compensation based on pre-measured channel responses is important, which can be done digitally by the frequency domain equalization technology [16].

(a)

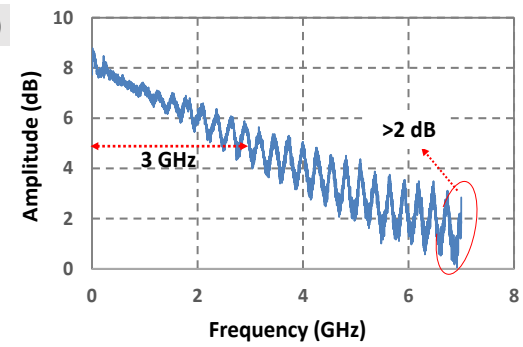

(b)

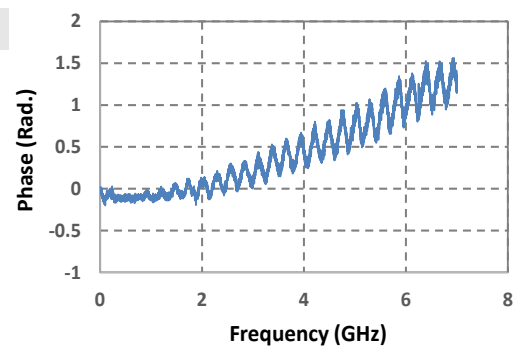

Fig. 3. Measured S21 curves for (a) amplitude and (b) phase response of the overall system.

\section{EXPERIMENTAL SETUP}

Figure 4 shows the experimental setup for the transmission of 112-Gb/s PAM-4 signal over 48.6-km SSMF within 50-GHz grid. At the transmitter, four external-cavity lasers (ECLs) spaced by $12.5 \mathrm{GHz}$ (in the $1550-\mathrm{nm}$ window) are used as the light sources, which are arranged in an odd and even pattern. The odd and even ECLs are combined by 3-dB optical couplers (splitters), respectively. Then, the combined outputs are fed into two Mach-Zehnder Modulator (MZM) and modulated independently. Both MZMs are biased at the quadrature point for linear modulation. The outputs of MZMs are combined by another 3-dB optical coupler, and then amplified using erbium doped fiber amplifier (EDFA). The inset of Fig. 4 shows the output spectral from the transmitter, where only $50-\mathrm{GHz}$ optical spectral is occupied for the 4 channels. Note that the adjacent channels are un-correlated in 
the setup. For transmitter DSP, a PAM-4 sequence (length: 32768 symbols) is first duobinary encoded. The encoded signal is pre-equalized digitally in the frequency domain by pre-measured S21 parameters. A 0.9 amplitude clipping (CLP) ratio is applied to reduce the peak to average power ratio (PAPR). The final signal is sent to a Tektronix arbitrary waveform generator (AWG) running at $14 \mathrm{GSa} / \mathrm{s}$. The RF signal from AWG is then filtered by a $5.5-\mathrm{GHz}(3 \mathrm{~dB})$ analog filter to further fit the signal in less than 12.5-GHz spectral. An electric amplifier is applied after the LPF to boost the driving amplitude. A feed-forward error correction (FEC) code of $7 \%$ overhead is assumed in the experiment with a BER threshold of $3.8 \times 10^{-3}$.

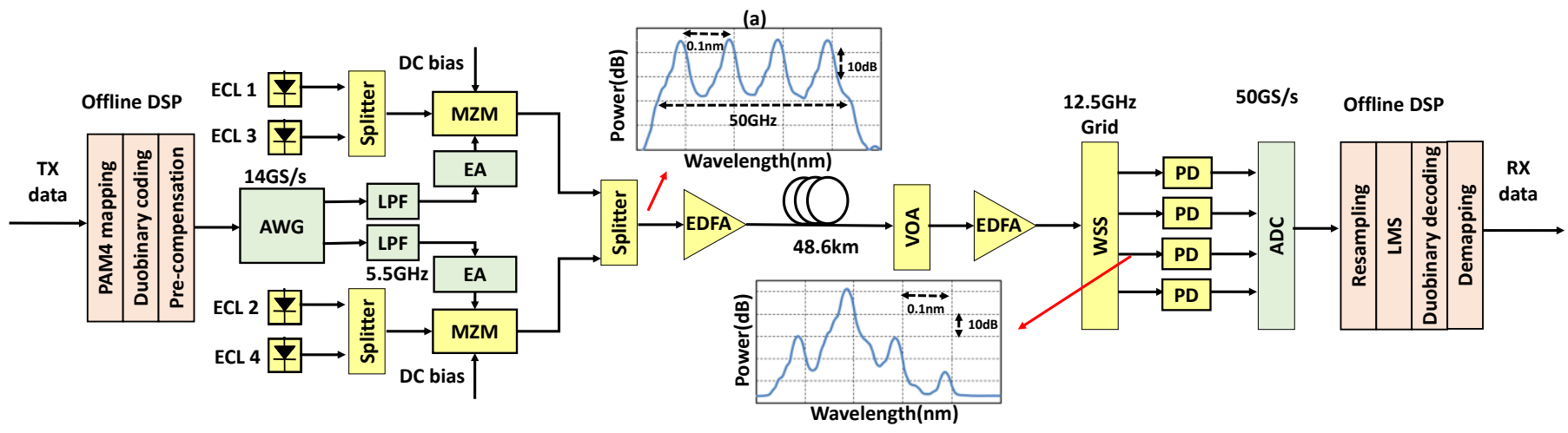

Fig. 4: Experimental setup for the transmission of 112(4x28)-Gb/s PAM-4 signal over 48.6-km SSMF within 50-GHz grid.

The transmission link is composed of a 48.6-km G.652 SMF (Dispersion: $17 \mathrm{ps} / \mathrm{nm} / \mathrm{km}$ ). The overall link loss budget is around $10 \mathrm{~dB}$. At the receiver, an EDFA is first used, which is followed by a wavelength selective switch (WSS) with 12.5-GHz channel spacing. The inset of Fig. 2 also shows the output spectral from WSS for the 2 nd channel. The filtered signals are detected by 40-GHz PIN-PD, respectively. The RF output of PD is then fed to a Tektronix real-time scope, acquired at $50 \mathrm{GSa} / \mathrm{s}$, and processed offline. In the receiver DSP, input data is first re-sampled to 2 sps, then TS-LMS is applied to restore the 7-level signal. The used number of taps in the TS-LMS algorithm is 47. Finally, PAM-4 signal is recovered by duobinary decoding, which is followed by PAM-4 de-mapping and BER calculation.

\section{RESUlTS AND DISCUSSIONS}

Figure 5 shows the measured receiver sensitivity for single-channel 28-Gb/s PAM-4 signal at back to back (with and without S21 pre-compensation). The maximum achievable receiver sensitivity is around $-19 \mathrm{dBm}$ with $7 \%$ FEC overhead, corresponding to a BER threshold of $3.8 \times 10^{-3}$. The inset of Fig. 5 also shows the restored eye diagram, when S21 pre-compensation is applied.

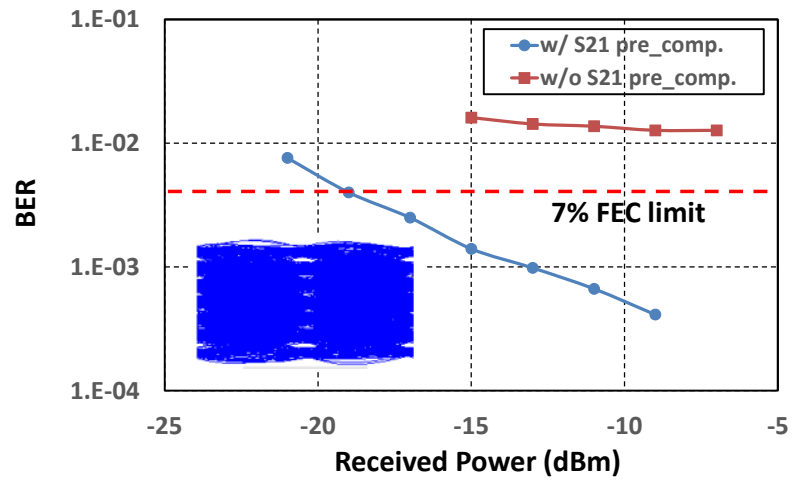

Fig. 5. Receiver sensitivity for single-channel 28-Gb/s signal, which is measured at back to back, with and without S21 pre-compensation.

A 7.2-GHz LPF is first used for spectrum shaping to Nyquist bandwidth. Two types of signal are under investigation, including 28-Gb/s PAM-4 and duobinary encoded PAM-4 (DB-PAM-4) signal. Both signals are recovered by the same TS-LMS algorithm in the receiver DSP. Fig. 6 shows the BER performance versus tap number of TS-LMS algorithm. It is observed that DB-PAM-4 signal requires much less number of taps than PAM-4 modulation, due to the pre-introduced inter-symbol interference (ISI) by duobinary encoding and 7-level TS-LMS algorithm. Result shows that the proposed method is more computationally efficient than PAM-4 modulation. 


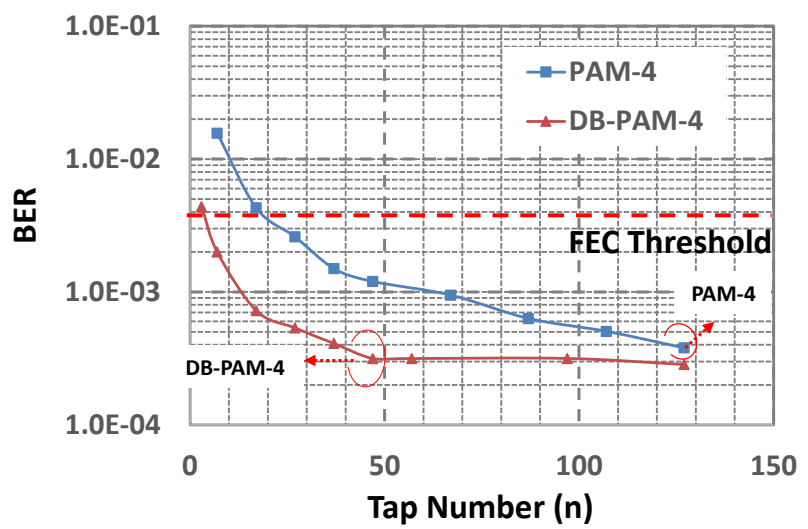

Fig. 6 BER performance versus number of taps used in TS-LMS algorithm for both 28-Gb/s PAM-4 and DB-PAM-4 signal under 7.2-GHz LPF.

Figure 7 shows the receiver sensitivity for both PAM-4 and duobinary PAM-4 signal at 28-Gb/s, under two types of LPF of 5.5 $\mathrm{GHz}$ and 7.2 GHz, respectively. Both signals are recovered by the TS-LMS algorithm. A 7.2-GHz analog LPF is first used for spectrum shaping at near Nyquist bandwidth. Results show that PAM-4 signal outperforms DB-PAM-4 slightly when the 3-dB bandwidth is larger than half of baud rate. However, in order to realize 4-channel 112-Gb/s transmission within only 50-GHz grid, each channel of the $28-\mathrm{Gb} / \mathrm{s}$ signal should occupy less than $12.5 \mathrm{GHz}$ optical spectral. Appling Nyquist filtering is not just enough, which will result in severe ICI. Then, 5.5-GHz LPF will satisfy such requirement with about 11-GHz 3-dB bandwidth for the shaped signal. Results show that PAM-4 signal cannot work with a 5.5-GHz LPF, while DB-PAM-4 does work. However, about 2-dB receiver sensitivity penalty is found compared to the 7.2-GHz case. The inset of Fig. 6 also shows the spectrum of 28-Gb/s DB-PAM-4 signal under 5.5-GHz and 7.2-GHz LPF, respectively.

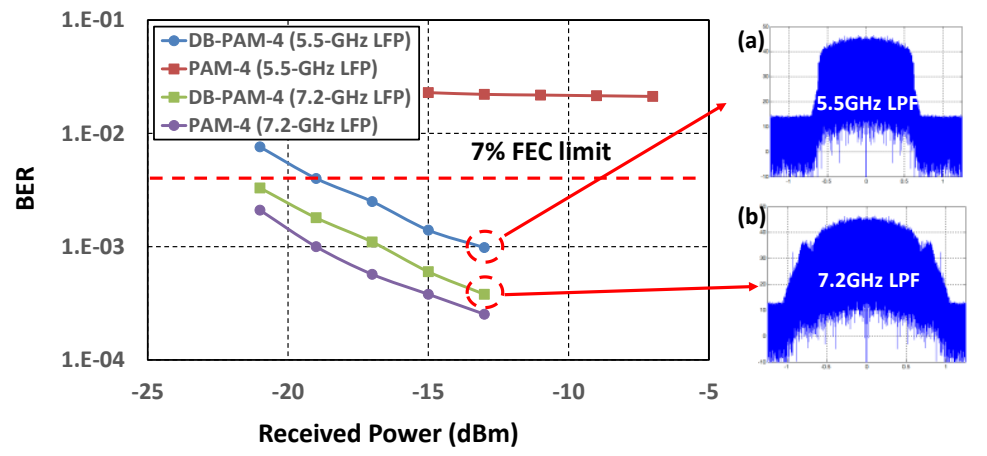

Fig. 7 Receiver sensitivity for both 28-Gb/s PAM-4 and duobinary PAM-4 signal, under two types of LPF of $5.5 \mathrm{GHz}$ and 7.2 GHz, respectively. (a) spectrum of DB-PAM-4 signal under 5.5GHz LPF, and (b) under 7.2GHz LPF.

Figure 8 shows the receiver sensitivity measured at back to back for both single channel $28-\mathrm{Gb} / \mathrm{s}$ and four-channel $112-\mathrm{Gb} / \mathrm{s}$ duobinary PAM-4 signal, in which 5.5-GHz LPF is used. The receiver sensitivity is about $-12 \mathrm{dBm}$ for the $112-\mathrm{Gb} / \mathrm{s}$ signal with $7 \%$ FEC overhead. About 7-dB sensitivity penalty is found, in which 6-dB penalty comes from the four times capacity increasing and $1-\mathrm{dB}$ penalty comes from the ICI.

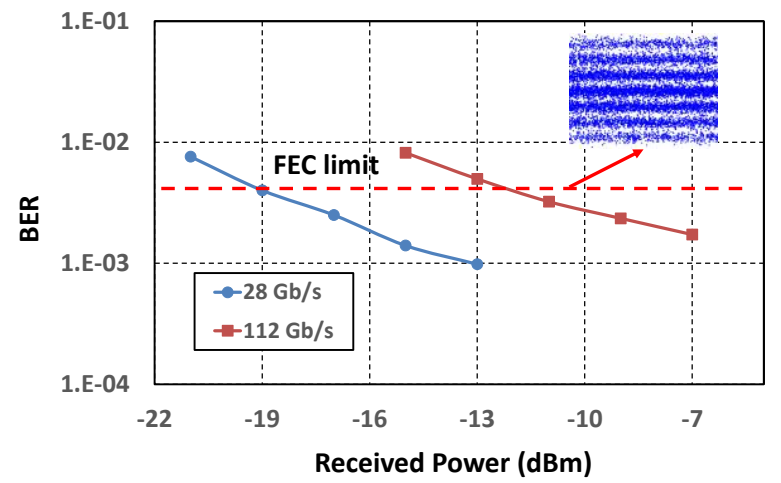

Fig. 8 Receiver sensitivity measured at back to back for both single channel 28-Gb/s and four-channel 112-Gb/s duobinary PAM-4 signal.

Finally, the receiver sensitivity is measured for four-channel 112-Gb/s duobinary PAM-4 signal over 48.6-km SSMF, as shown in Fig. 9. Less than 2-dB sensitivity penalty is found over 48.6-km SSMF, thanks to the narrowed signal bandwidth by duobinary 
filtering and LPF.

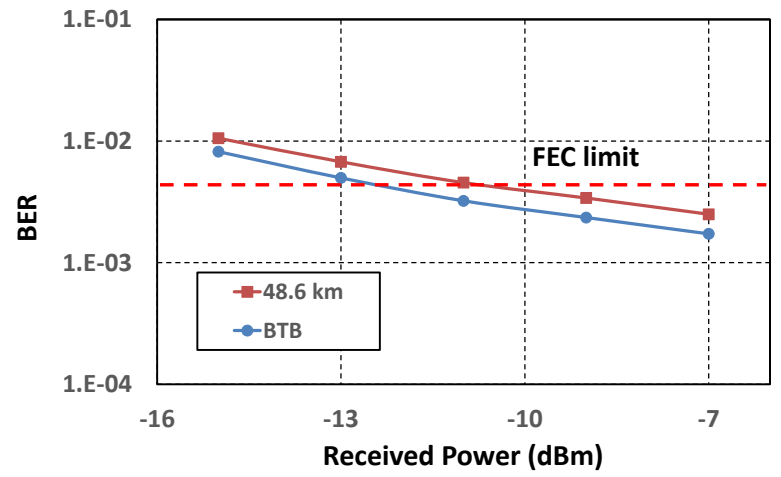

Fig. 9 Receiver sensitivity measured for four-channel 112-Gb/s duobinary PAM-4 signal within 50-GHz grid over 48.6-km SSMF.

\section{CONCLUSIONS}

A transmission of 112(4x28)-Gb/s PAM-4 signal over 48.6-km SSMF has been experimentally demonstrated within only 50-GHz grid, achieving an optical SE of $2.24 \mathrm{~b} / \mathrm{s} / \mathrm{Hz}$. This is the highest SE to the best of our knowledge that has been reported for the intensity modulation and direct detection based PAM-4 transmission. The proposed method has been shown bandwidth and computationally efficient, which is thought to be feasible in the low-cost short reach optical networks.

\section{Acknowledgement}

This work was supported in part by the China National Science Foundation Project (61505154), in part by the National Science Foundation of China under Grant No.61201086, in part by the China Scholarship Council under Grant No.201506375060, in part by the Planned Science and Technology Project of Guangdong Province under Grant 2013B090500007, and in part by the Dongguan Project on the Integration of Industry, Education and Research under Grant 2014509102205.

\section{REFERENCES}

[1] K. Zhong, X. Zhou, T. Gui, L. Tao, Y. Gao, W. Chen, and C. Lu, "Experimental study of PAM-4, CAP-16, and DMT for $100 \mathrm{~Gb} / \mathrm{s}$ short reach optical transmission systems," Opt. Exp. 23(3), 1176-1189 (2015).

[2] M. Chagnon, M. Osman, M. Poulin, C. Latrasse, J. F. Gagné, Y. Painchaud, and D. Plant, "Experimental study of $112 \mathrm{~Gb} / \mathrm{s} \mathrm{short} \mathrm{reach} \mathrm{transmission} \mathrm{employing}$ PAM formats and SiP intensity modulator at $1.3 \mu$ m," Opt. Exp. 22(17), 21018-21036 (2014).

[3] M. I. Olmedo, T. Zuo, J. B. Jensen, Q. Zhong, X. Xu, S. Popov, and I. T. Monroy, "Multiband carrierless amplitude phase modulation for high capacity optical data links," J. Lightwave Technol. 32(4), 798-804 (2014).

[4] J. C. Cartledge, and A. S. Karar, "100 Gb/s intensity modulation and direct detection," J. Lightwave Technol. 32(16), 2809-2814 (2014).

[5] D. Sadot, G. Dorman, A. Gorshtein, E. Sonkin, and O. Vidal, "Single channel 112Gbit/sec PAM4 at 56Gbaud with digital signal processing for data centers applications," Opt. Exp. 23(2), 991-997 (2015).

[6] J. Zhang, J. Yu, F. Li, X. Li, and Y. Wang, "Demonstration of Single-Carrier ETDM 400GE PAM-4 Signals Generation and Detection." IEEE Photonics Technol. Lett. 27(24), 2543-2546 (2015).

[7] L. Zhang, E. Zhou, Q. Zhang, G. N. Liu, and T. Zuo, "C-band Single Wavelength 100-Gb/s IM-DD Transmission over 80-km SMF without CD compensation using SSB-DMT," in Proc. OFC'15, paper. Th4A.2 (2015).

[8] Y. Kai, M. Nishihara, T. Tanaka, R. Okabe, T. Takahara, J. C. Rasmussen, and K. Ogawa, "130-Gbps DMT transmission using silicon Mach-Zehnder modulator with chirp control at 1.55- $\mu$ m," in Proc. OFC'15, paper. Th4A.1 (2015).

[9] R. Hirai, H. Toyoda, and N. Kikuchi, "Proposal of new 400GbE signaling formats with $4 \lambda \times 100 \mathrm{G}$ configuration," in contribution to IEEE $802.3400 \mathrm{~Gb} / \mathrm{s}$ Ethernet Study Group, Interim Meeting, Indian Wells, USA, Jan. 2014.

[10] H. Zhang, S. Fu, J. Man, W. Chen, X. Song, and L. Zeng, "30km downstream transmission using 4× 25Gb/s 4-PAM modulation with commercial 10Gbps TOSA and ROSA for 100Gb/s-PON," in Proc. OFC'15, paper. M2I.3 (2014).

[11] C. Chen, X. Tang, and Z. Zhang, "Transmission of 56-Gb/s PAM-4 over 26-km single mode fiber using maximum likelihood sequence estimation," in Proc. OFC'15, paper. Th4A.5 (2015).

[12] N. Kikuchi, R. Hirai, and T. Fukui, "Practical implementation of 100-Gbit/s/lambda optical short-reach transceiver with Nyquist PAM4 signaling using electroabsorptive modulated laser (EML)," in Proc. OFC'15, paper. Th3A.2 (2015).

[13] A. Lender, "Correlative digital communication techniques," IEEE Trans. Commun. Technol. 12(4), 128-135 (1964).

[14] X. Zhou, J. Yu, M.-F. Huang, Y. Shao, T. Wang, P. Magill, M. Cvijetic, L. Nelson, M. Birk, G. Zhang, S. Ten, H. B. Matthew, and S. K. Mishra, "Transmission of 32- Tb/s capacity over $580 \mathrm{~km}$ using RZ-shaped PDM-8QAM modulation format and cascaded multimodulus blind equalization algorithm," J. Lightwave Technol., 28(4), 456-465, 2010.

[15] L. Suhr, J. V. Olmos, B. Mao, G. N. Liu, and I. T. Monroy, "Direct modulation of 56 Gbps duobinary-4-PAM." in Proc. OFC'15, paper. Th1E.7 (2015).

[16] D. Falconer, S. L. Ariyavisitakul, A. Benyamin-Seeyar and B. Eidson, "Frequency domain equalization for single-carrier broadband wireless systems," IEEE Commun Magazine, vol. 40, no. 4, pp. 58-66, Apr. 2002. 